\title{
Auditoria de gestão e estratégia no setor público
}

\author{
Carlos Alberto Sampaio de Freitas
}

\section{Introdução}

A auditoria de gestão é uma modalidade da auditoria de resultados que analisa as estruturas, os sistemas e as práticas gerenciais de uma organização ou de um programa, com a finalidade de oferecer subsídios à melhoria desses elementos, partindo do pressuposto que a referida melhoria propiciará o aumento do nível de eficácia, eficiência e efetividade do auditado.

O objetivo do presente artigo, além de evidenciar a importância da auditoria de gestão para o fortalecimento da capacidade gerencial do setor público, é o de tentar construir um arcabouço lógico que abrigue todas as possíveis dimensões que podem ser exploradas em um trabalho dessa espécie, voltado especificamente para a gestão estratégica de organizações e programas públicos.

Além do aspecto teórico, são listadas perguntas que podem ser utilizadas como pontos de partida em uma investigação nessa modalidade de auditoria, além de exemplos práticos de situações em que são encontrados os tipos de falhas que a auditoria de gestão pode evidenciar e ajudar a eliminar.

Segundo Grateron:

"a auditoria de gestão é uma técnica ou atividade que presta consultoria aos mais altos estratos de uma organização, seja de caráter público ou privado. Atualmente o ritmo das mudanças tem sido acelerado e contínuo, o que exige das organizações ajustes nas estratégias para enfrentar o desconhecido e garantir o alcance de suas metas e objetivos. Esse ritmo e a velocidade destas mudanças
Carlos Alberto Sampaio de Freitas é especialista em Avaliação de Políticas Públicas pela Universidade do Rio de Janeiro (UFRJ) e Analista de Finanças e Controle do Tribunal de Contas da União.

Contato: carlossf@ tcu.gov.br 
variam de um setor para outro dependendo de inúmeras condicio-

nantes externas e internas. A auditoria de gestão objetiva melhorar a capacidade da organização para reagir com sucesso às mudanças, partindo da equipe diretiva, e procura auxiliar a Diretoria na avaliação interna da organização e de seus executivos."

Essa modalidade de auditoria procura mostrar os pontos fracos e fortes da organização, estabelecendo as recomendações necessárias para melhorar o processo de tomada de decisões. Compreende os objetivos estratégicos da organização examinada e, por isso, analisa a estrutura da organização, as linhas de controle e comunicação, o fluxo de informações e a susceptibilidade de se adaptar às mudanças de estratégia para atingir os objetivos desejados ou planejados. Está dirigida para o futuro da organização.

Nessa linha, fica evidente que ela é fortemente dependente do paradigma gerencial vigente, uma vez que os julgamentos efetuados pelos auditores estarão baseados em premissas do modelo vigente de gerenciamento público.

É desnecessário, portanto, enfatizar a importância de um profundo conhecimento das teorias e das práticas administrativas aplicáveis ao setor público.

\section{Gestão estratégica orientada para resultados}

A constatação dos meios acadêmicos é a de que o Estado, antes governado exclusivamente por um paradigma burocrático, está passando por reformas que têm como um dos paradigmas o gerencial.

$\mathrm{O}$ ponto de vista gerencial enfatiza que o controle, antes realizado em função de padrões de conformidade a regras, leis e regulamentos, passe a ser efetuado em cima dos resultados efetivamente produzidos pelos gerentes e organizações públicas.

Nesse contexto, o gerenciamento pelos resultados (performance management) tornou-se a peça-chave para reformas realizadas em diversos países.

Mas o que vem a ser gerenciamento pelos resultados ou performance management?

Pode-se definir performance management como um processo de gerenciamento resultante da reunião das práticas de mensuração de resultados que vinham se desenvolvendo (performance measurement) com as práticas governamentais de planejamento estratégico (strategic management). 
Seus componentes básicos são:

- estratégia;

- missão;

- objetivos estratégicos;

- metas;

- indicadores de desempenho;

- monitoramento; e

- ações corretivas.

Esses elementos interagem com a estrutura organizacional, os recursos humanos e as instalações e equipamentos em um processo que pode ser representado na forma da figura abaixo:

\section{Performance management}

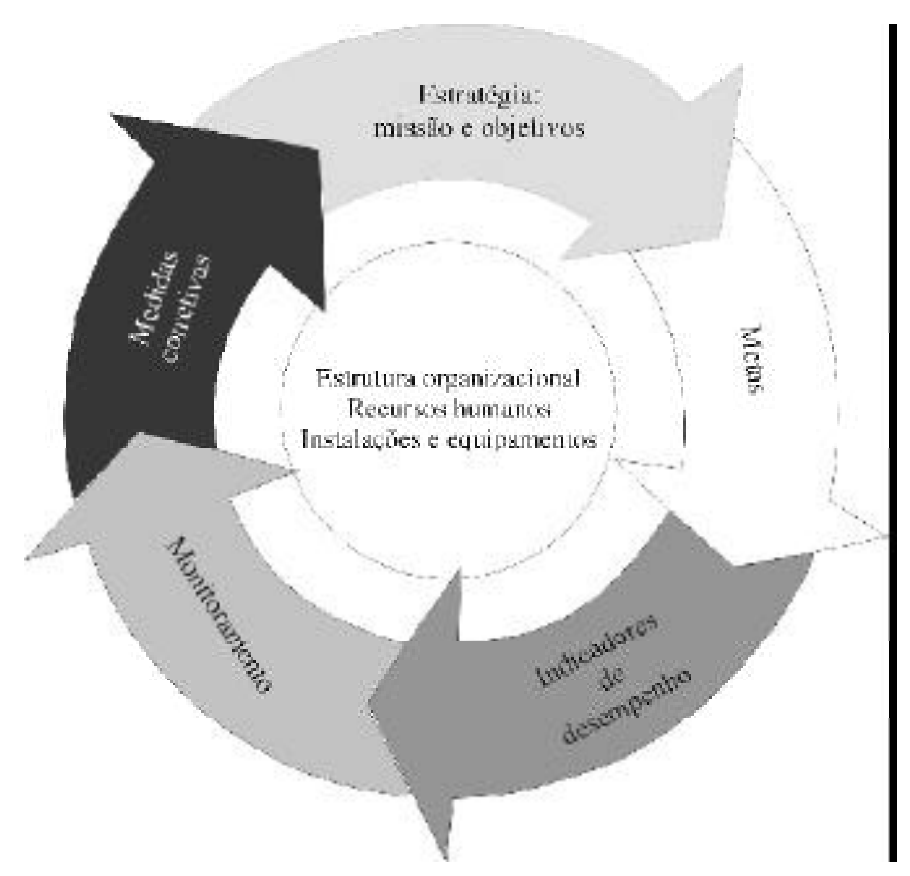

A auditoria de gestão é parte integrante do monitoramento, ao lado de outras práticas de mensuração de resultados, tais como a avaliação de programas, auditoria de desempenho, análise de risco etc.

É importante ressaltar que a responsabilidade primária pelo monitoramento é do próprio gestor, uma vez que as informações fornecidas acerca dos resultados devem desempenhar um papel fundamental no processo decisório das organizações públicas.

Assim, ao direcionar os exames para um enfoque predominantemente sistêmico, estar-se-á oferecendo aos próprios gestores dos órgãos 
ou entidades auditados indicativos relacionados a aspectos gerenciais e operacionais que podem e devem ser melhorados.

\section{Dentro desse contexto, como agregar valor por intermédio da auditoria de gestão?}

Para efeito didático, vamos explorar diferentes possibilidades, formulando questões relativas aos elementos essenciais para a gestão estratégica orientada para resultados.

\section{Missão}

Segundo Rosseto, “a missão é composta pelo propósito e pelos valores básicos da organização, bem como seu escopo de operações. Constitui a declaração básica da razão da existência da organização", relacionando a organização pública com a capacidade de satisfazer uma necessidade pública.

A verificação da missão visa:

"certificar-se quanto a alguns requisitos essenciais que determinam a própria razão da continuidade operacional e institucional dos Órgãos ou Entidades da Administração Pública Federal (pois o sentido maior da existência — e continuidade — dos Órgãos/Entidades somente se justifica quando estes têm como apresentar evidências que justifiquem esta continuidade, o que é materializado pelos tipos de resultados que conseguem oferecer à sociedade e o nível de aderência de suas operações, como um todo, às efetivas necessidades/fundamentos que determinaram a sua criação)."

(Secretaria Federal de Controle Interno do Ministério da Fazenda).

Pode ser que a missão de uma organização esteja mal formulada, em função de uma estruturação inadequada do problema para o qual ela foi criada para resolver. Outra possibilidade é que o problema esteja bem estruturado, porém a declaração da missão esteja incorreta, seja por uma abordagem muito abrangente, muito restrita ou simplesmente equivocada. Por último, o problema pode ter sido corretamente atacado e a missão originalmente ter sido bem formulada; porém, ao longo do tempo, as circunstâncias para as quais aquela missão foi formulada simplesmente mudaram, o que não é incomum ocorrer em um ambiente cada vez mais mutável.

Outra ocorrência muito comum no setor público é a superposição de missões, atividades e programas de diferentes órgãos e entidades, situação que diminui a eficácia, a eficiência e a efetividade dos serviços públicos.

Um outro caso a ser considerado é a não-concretização da missão do órgão ou entidade. Nesse caso, as causas deverão ser buscadas pelo 
auditor, a fim de que possam ser sugeridas medidas que possibilitem o cumprimento das finalidades do órgão.

Assim, as perguntas que orientam o planejamento da auditoria podem ser formuladas nos seguintes termos:

a) Qual é a missão do auditado?

b) A missão está corretamente formulada?

c) A missão do auditado é adequada ou perdeu o valor público?

d) O auditado está cumprindo a missão?

e) Há superposição de missões ou funções com outras organizações?

\section{Estratégia}

A estratégia é uma ferramenta gerencial usada para ajudar a organização a executar melhor o seu trabalho, para focalizar a sua energia e para assegurar que os membros da organização estejam trabalhando na busca dos mesmos objetivos, disciplinando as decisões fundamentais e as ações que a guiam com foco no futuro.

Conceitualmente, ela deve:

- partir de uma missão ou propósito;

- demonstrar os recursos ou meios que serão empregados na sua consecução; e

- detalhar como deve ser estruturada e operada a organização para a consecução dos objetivos.

Para que uma estratégia tenha alguma possibilidade de êxito, é preciso, em primeiro lugar, que ela seja bem formulada. Isso posto, a elaboração de qualquer plano estratégico no setor público deve partir da análise do ambiente externo, buscando oportunidades e ameaças não para a existência da organização, mas sim para a realização de sua missão e para a geração de valor público, bem como do ambiente interno, identificando os pontos fortes e fracos da organização.

Segundo Moore, qualquer estratégia corporativa do setor público deve ser questionada em três aspectos:

- produz valor público (gera resultados);

- tem legitimidade política suficiente para se sustentar; e

- é administrativa e operacionalmente viável.

Ainda que bem formulada, as possibilidades de fracasso de uma estratégia são enormes, visto que a maior dificuldade está na sua implementação, estimando-se que a taxa de insucesso varia de $70 \%$ a 90\% (Kaplan e Norton, 2001).

Nesse ponto, entram em cena os objetivos estratégicos, peças-chave para a execução bem-sucedida de uma estratégia. Um complicador adicional é que, no setor público, eles tendem a ser um tanto vagos, como resultado da disputa das diversas prioridades políticas que competem entre 
si em uma organização governamental e das diferentes visões dos stakeholders dos programas.

Esses objetivos estratégicos devem estar alinhados com as diretrizes governamentais e com a missão da organização, concorrendo para sua realização. Também devem possibilitar o estabelecimento de metas ou indicadores de desempenho mensuráveis, de maneira que o desempenho da organização (em função da estratégia adotada) possa ser monitorado.

\section{Estratégia: principais elementos}

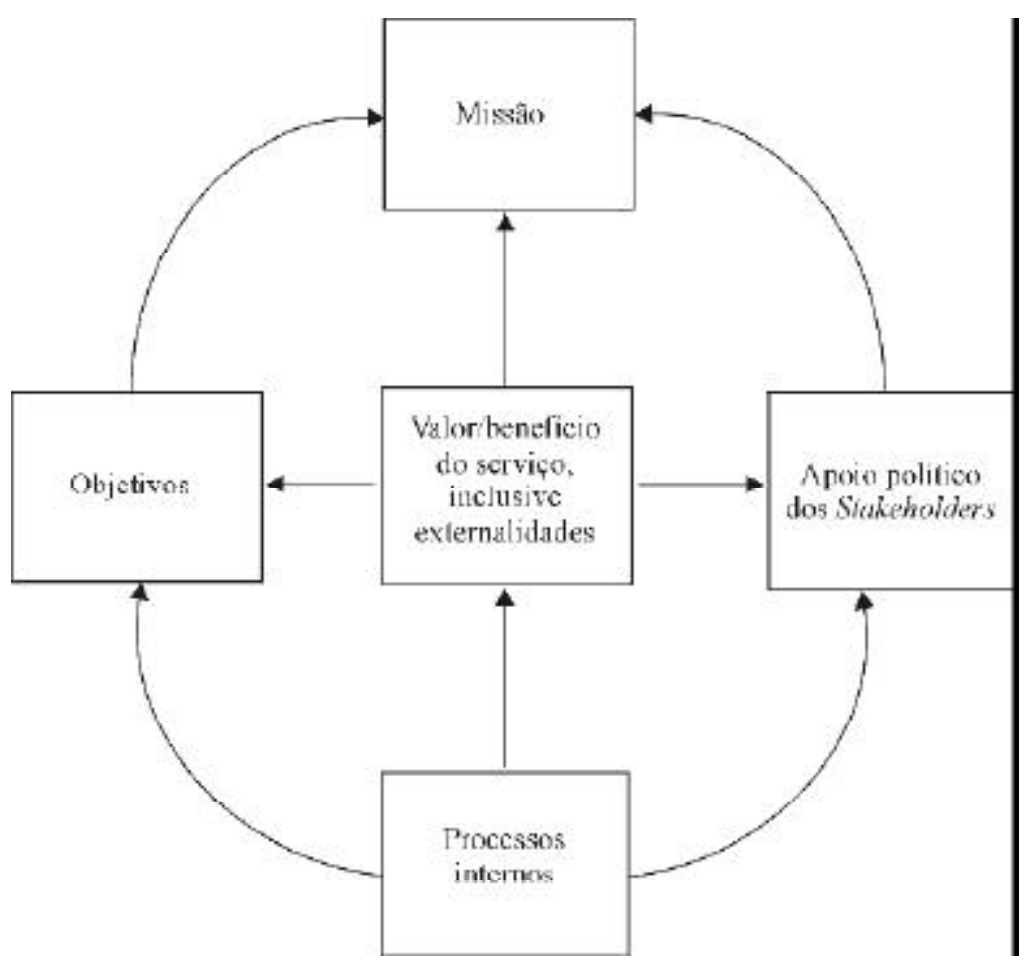

Portanto, as perguntas que orientam o planejamento da auditoria podem ser as seguintes:

a) Os objetivos estratégicos estão alinhados com a missão?

b) Estão alinhados com as prioridades do governo?

c) Foram identificados pontos fortes e fracos da organização? Quais?

d) Os objetivos estratégicos levaram em consideração os pontos fortes e fracos?

e) Os objetivos estratégicos estão suficientemente claros, sem ambigüidades? 


\section{Metas organizacionais e indicadores de desempenho}

As metas e os indicadores de desempenho fornecem o instrumental para a mensuração do desempenho da organização (em diversos níveis) ou programa.

Medir o desempenho envolve a determinação de um conjunto de indicadores relativos à organização como um todo e às suas divisões, processos, atividades etc., que devem refletir os resultados de cada nível observado.

Mas, como garantir que esse sistema de mensuração contribua para o sucesso da organização ou do programa?

Em primeiro lugar, é fundamental que esses indicadores estejam alinhados com a estratégia da organização ou esta última não se materializará, em razão da dispersão de esforços ou da falta de focalização de esforços.

As medidas de desempenho devem derivar da declaração de missão e da estratégia organizacional, instrumentalizando o monitorameto dessa última em todos os níveis e demonstrando aos seus membros como o seu trabalho e desempenho são importantes para o sucesso da organização.

Os princípios fundamentais de um sistema de medição de desempenho também devem ser observados:

- medir somente o que é importante;

- equilibrar um conjunto de medidas que cubram diferentes dimensões dos resultados (eficácia, eficiência, efetividade, qualidade e produtividade);

- medir recursos e resultados;

- envolver os funcionários no desenho e na implementação do sistema de mensuração; e

- alinhar as medidas com os objetivos e as estratégias organizacionais.

Como um processo, a medição do desempenho não se refere, simplesmente, a uma coleta de dados associada a um objetivo predefinido. As melhores medidas de desempenho são como um sistema de alerta, que envolve prevenção e identificação, direcionado à adequação dos produtos e/ou serviços ao uso e ao aumento da satisfação dos clientes.

Adicionalmente, as medidas de desempenho e o gerenciamento de processos estão focalizados no incremento da eficiência e efetividade dos processos, produtos e/ou serviços, pela sua otimização, dentro de um ciclo contínuo que monitora, identifica e captura as oportunidades de melhoria desses processos, produtos e/ou serviços, por meio da implementação de melhores práticas.

As medidas de desempenho também devem possuir alguns atributos, quais sejam: 
- refletir tanto as necessidades dos clientes quanto as da organização;

- fornecer uma base adequada para a tomada de decisões;

- ser de fácil compreensão;

- ter ampla aplicação;

- ser interpretada uniformemente;

- ser possível de ser mensurada com os sensores (informações) existentes;

- ser precisa na interpretação dos dados;

- ser economicamente aplicável;

- confiabilidade;

- validade;

- relevância; e

- consistência.

Em síntese, as medidas de desempenho possibilitam:

- identificar se as necessidades dos clientes estão sendo atendidas;

- revelar problemas e identificar oportunidades de melhoria; e

- mostrar se as melhorias estão acontecendo.

Por último, é importante ressaltar que toda medida de desempenho deve estar vinculada a uma meta ou padrão predefinido, pois é a única forma de interpretar, significativamente, os resultados das medidas e estimar o sucesso de um sistema administrativo.

Além dos aspectos já mencionados, as perguntas que devem nortear a auditoria de gestão no que se refere às metas e aos indicadores de desempenho são as seguintes:

\section{Metas}
a) Quais são?
b) Estão alinhadas com a estratégia?
c) Foram cumpridas? Se negativo, identificar as causas.
d) As metas são claras e relevantes?
e) O processo de definição das metas é bem fundamentado?
f) As metas são relativas às atividades-chave?
g) As metas são periodicamente revisadas?
h) Quais as conseqüências do não-cumprimento das metas?
i) Medidas são adotadas quando as metas não são cumpridas?

\section{Indicadores de desempenho}
a) Existem?
b) Estão alinhados com a estratégia, objetivos e metas?
c) Como se dá o monitoramento?
d) O sistema de monitoramento dos resultados é completo? 
e) Os indicadores são relevantes, confiáveis etc.?

f) A relação custo-benefício é adequada?

g) Os indicadores são utilizados para alguma finalidade ou apenas como uma formalidade burocrática?

\section{Estrutura organizacional}

A estrutura organizacional está intimamente relacionada com os sistemas de gerenciamento, afetando em maior ou menor escala os sistemas de informação, o processo decisório, a cultura etc.

No setor público, quanto maior o nível hierárquico, maior o componente político da estrutura, que funciona com um sistema de equilíbrio ou distribuição de poder.

Existem diferentes possibilidades de organização, cujas vantagens e desvantagens genéricas são expostas a seguir:

\section{Funcional}

\begin{tabular}{l|l}
\hline Vantagens & Desvantagens \\
\hline • Excelente coordenação & $\bullet$ Conflitos interdepartamentais \\
intradepartamental & de especialistas \\
- Especialização & $\bullet$ Fragmentação do processo \\
• Baixo custo administrativo & decisório \\
& $\bullet$ Subobjetivação \\
\hline
\end{tabular}

\section{Geográfica}

\begin{tabular}{|c|c|}
\hline Vantagens & Desvantagens \\
\hline $\begin{array}{l}\text { - Foco no cliente } \\
\text { (customização do serviço) }\end{array}$ & - Duplicidade de recursos \\
\hline - Favorece a descentralização & $\begin{array}{l}\text { - Dificuldades na coordenação } \\
\text { e controle }\end{array}$ \\
\hline
\end{tabular}

\section{Por clientes}

\begin{tabular}{l|l}
\hline Vantagens & Desvantagens \\
\hline - Satisfação dos clientes & $\bullet$ Superposição de recursos \\
- Facilidade para identificar & $\bullet$ Complexidade organizacional \\
necessidades & \\
• Possibilidade de mudanças & \\
mais rápidas & \\
\hline
\end{tabular}


Por produtos ou serviços

\begin{tabular}{l|l}
\hline Vantagens & Desvantagens \\
\hline • Foco no produto & $\bullet$ Duplicidade de recursos \\
- Melhor qualidade & $\bullet$ Mensuração de resultados \\
- Melhor mensuração de & mais difícil (outcomes) \\
resultado (output) & \\
\hline
\end{tabular}

\section{Divisional ou por projetos}

\begin{tabular}{l|l}
\hline Vantagens & Desvantagens \\
\hline - Inovação e flexibilidade & $\bullet$ Capacidade ociosa de recursos \\
• Possibilidade de direcionamento & materiais e humanos \\
dos recursos & $\begin{array}{c}\bullet \text { Instabilidade na formação } \\
\text { de grupo }\end{array}$ \\
$\begin{array}{l}\text { Equipe e gerência altamente } \\
\text { adminitadas técnica e }\end{array}$ & \\
\hline
\end{tabular}

\section{Matricial}

\begin{tabular}{l|l}
\hline Vantagens & Desvantagens \\
\hline • Maximização das vantagens & $\bullet$ Confusão \\
da estrutura funcional & $\bullet$ Conflito e tensão \\
- Maximização das vantagens da & $\bullet$ Indefinição de responsabilidades \\
estrutura por produtos & \\
- Minimização das desvantagens & \\
das duas estruturas & \\
\hline
\end{tabular}

\section{Processo de organização}

Para se chegar a qualquer desses arranjos, é necessário estruturar um processo lógico-analítico, que pese todos os prós e contras, e que permita determinar a melhor forma de departamentalização.

Segundo Chiavenato, os quatro passos básicos para organizar são:

\section{a) Divisão do trabalho}

Dividir a carga de trabalho em tarefas que possam ser logicamente executadas por indivíduos ou grupos. 
Combinar e agrupar tarefas e pessoas de maneira lógica e eficiente em unidades organizacionais.

\section{c) Hierarquia}

Especificar quem deve se subordinar a quem na organização.

\section{d) Coordenação}

Estabelecer mecanismos para integrar as unidades departamentais em um todo coerente e monitorar a eficácia dessa integração.

Assim, segundo o mesmo autor, o propósito principal do desenho organizacional é colocar a estrutura a serviço do ambiente, da estratégia, da tecnologia, das pessoas e das atividades desenvolvidas, articulando os recursos para servir à missão e aos objetivos principais.

Em termos de auditoria de gestão, interessa saber se a estrutura favorece (ou pelo menos não prejudica) a implementação da estratégia da organização e o atingimento dos seus objetivos, buscando identificar possíveis problemas do arranjo estrutural da entidade auditada.

As possíveis indagações a serem respondidas em relação à estrutura organizacional são as seguintes:

a) É adequada aos objetivos do órgão?

b) No caso da descentralização de programas ou atividades, existem sistemas de monitoramento que permitam monitorá-los eficazmente?

c) Existem contradições entre a departamentalização e a estratégia?

d) Há adequada definição de responsabilidades?

e) Considerando as especificidades da organização auditada, as vantagens do tipo de departamentalização adotado superam as desvantagens?

\section{Recursos humanos}

Em última instância, em uma organização, são as pessoas que executam as tarefas, elaboram a estratégia, fornecem os serviços etc.

A dimensão dos recursos humanos é bastante ampla, envolvendo aspectos de motivação, liderança, cultura etc. Alguns desses aspectos não estão compreendidos no escopo da auditoria de gestão ora proposto.

Interessa explorar, por enquanto, os aspectos dos recursos humanos mais diretamente relacionados à implementação da estratégia e dos objetivos organizacionais.

Sob esse prisma, os possíveis questionamentos seriam do tipo:

a) São suficientes (em termos quantitativos) para o cumprimento da missão, estratégia, objetivos e metas da organização? 
metas?

b) Estão capacitados para o cumprimento da missão, objetivos e

c) O sistema de recompensas explícito e/ou implícito favorece a implementação da estratégia?

d) $\mathrm{O}$ ferramental de mensuração de resultados é entendido como um auxílio ao trabalho ou, ao contrário, uma tarefa a mais a ser cumprida (que não agrega valor) ou ainda, um simples aparato de controle?

\section{Instalações, equipamentos e recursos financeiros}

Sem uma adequada infra-estrutura, não se consegue levar adiante qualquer estratégia ou obter resultados satisfatórios, por isso, é importante comparar os resultados obtidos aos recursos efetivamente empregados, indagando se:

a) São suficientes (em termos quantitativos) para o cumprimento da missão, estratégia, objetivos e metas?

b) Houve contingenciamento orçamentário que dificultasse o cumprimento das metas?

c) As instalações são adequadas às atividades desenvolvidas pelo órgão ou provocam algum efeito negativo nos resultados?

d) Há algum tipo de efeito na distribuição de instalações, equipamentos e recursos financeiros que atue negativamente nos resultados?

\section{Conclusão}

Como vimos, a auditoria de gestão é uma poderosa ferramenta para a melhoria da ação governamental, uma vez que ela fornece informações e análises precisas sobre o estágio de gerenciamento do setor público; ajuda a sintonizar as ações da organização pública com sua missão; evidencia discrepâncias entre os resultados reais e os informados pelo órgão; recomenda procedimentos que melhorem o processo de elaboração de metas e indicadores de desempenho, além de contribuir para o aumento da capacidade de mudança e inovação das organizações públicas. Sua utilização sistemática, não só por entidades de controle governamental, mas pelo próprio gestor público, tem um considerável potencial de alavancagem das boas práticas gerenciais e de auxílio na construção de uma estrutura de gestão mais consistente nas organizações públicas. 


\section{| Referências bibliográficas}

Batista, Fábio Ferreira. (1999), Curso "Elaboração de Indicadores de Desempenho Institucional". Instituto Serzedello Corrêa. Mimeo. Brasília.

Chiavenato, Idalberto. (1999), Administração nos novos tempos: os novos horizontes em administração. São Paulo: Makron Books.

FreItAs, Carlos Alberto Sampaio de. (1999), "A implementação do Government Performance and Results Act na administração pública dos EUA". Revista do Serviço Público. Brasília, ENAP, v.50, n.3, 93-122.

Galbraith, Jay R. (1977), Organization Design. Massachussetts: Addison Wesley.

Grateoni, Ivan Ricardo Guevara. (1999), "Auditoria de Gestão: utilização de indicadores de gestão no setor público". Dissertação apresentada à Faculdade de Administração, Economia e Contabilidade da Universidade de São Paulo, para obtenção do título de Mestre em Contabilidade e Controladoria. São Paulo.

Kaplan, Robert S., NoRTon, David P. (2000), Organização orientada para a estratégia: como as empresas que adotam o balanced scorecard prosperam no novo ambiente de negócios. Rio de Janeiro: Campus.

National Audit Office. (1998), Performance measurement: what to look for in value for money studies. London. . (2001), Measuring the Performance of Government Departaments.

London.

Moore, Mark Harrison. (1998), Creating Public Value: strategic management in government. Harvard University Press.

Secretaria Federal de Controle. Programa de Auditoria: auditoria de missão. Distrito Federal.

Swiss, James Edwin. (1991), Public Management systems:monitoring and managing government performance. New Jersey: Prentice-Hall International.

Tachizawa, Takeshy; Scaico, Oswaldo. (1997), Organização Flexível: qualidade na gestão por processos. São Paulo: Editora Atlas.

United States Accounting Office. (1999), Strengthening Regulatory Agencies'Performance Management Practices. Washington, DC.

Universidade Federalde Santa Catarina. (2000), Curso de Extensão em Gestão Estratégica de Projetos. Florianópolis, Santa Catarina. 


\section{Auditoria de gestão e estratégia no setor público \\ Carlos Alberto Sampaio de Freitas}

Auditoria de gestão é uma modalidade da auditoria de resultados que analisa as estruturas, os sistemas e as práticas gerenciais de uma organização ou de um programa, com a finalidade de oferecer subsídios à melhoria desses elementos, partindo do pressuposto que a referida melhoria propiciará o aumento do nível de eficácia, eficiência e efetividade do auditado.

O objetivo do presente artigo, além de evidenciar a importância da auditoria de gestão para o fortalecimento da capacidade gerencial do setor público, é o de tentar construir um arcabouço lógico que abrigue todas as possíveis dimensões que podem ser exploradas em um trabalho dessa espécie, voltado especificamente para a gestão estratégica de organizações e programas públicos.

Além do aspecto teórico, são listadas perguntas que podem ser utilizadas como pontos de partida em uma investigação dessa modalidade de auditoria, abrangendo a missão, estratégia, metas e indicadores de desempenho, estrutura organizacional, recursos humanos e instalações, equipamentos e recursos financeiros, elementos cujo alinhamento é essencial para uma gestão estratégica voltada para resultados.

\section{Auditoría de gestión y estrategia en el sector público \\ Carlos Alberto Sampaio de Freitas}

Auditoría de gestión es una modalidad de auditoría de resultados que analiza las estructuras, los sistemas y las prácticas gerenciales de una organización o de un programa, con la finalidad de ofrecer subsidios para mejorar eses elementos, tiendo como supuesto que esa mejora propiciará mayor grado de eficacia, eficiencia y efectividad del auditado.

El objetivo de este artículo, además evidenciar la importancia de la auditoría de gestión para el fortalecimiento de la capacidad gerencial del sector público, es experimentar construir un soporte lógico que incluya todas las posibles dimensiones que pueden ser exploradas en trabajo de esa naturaleza, mirando especificamente la gestión estratégica de organizaciones y programas públicos.

Además el aspecto técnico, son listadas preguntas que pueden ser utilizadas como puntos de partida en una investigación de esa modalidad de auditoría, contenendo la misión, estrategia, metas e indicadores de desempeño, estructura organizacional, recursos humanos e instalaciones, equipamentos y recursos financieros, elementos cuyo alineamiento es esencial para una gestión estratégica con fines a obtener resultados.

\section{Management audit and strategy in the public sector \\ Carlos Alberto Sampaio de Freitas}

Management audit is an audit of results, modality that analyzes the structures, the systems and the managerial practices of an organization or program with the purpose of offering subsidies to their improvement, with the premisse that this improvement will increase the level of economy, efficiency and effectiveness of the auditee.

The objective of the present article, besides evidencing the importance of the management audit for the strengthening of the managerial capacity of the public section, is to build a logical framework that approaches all the possible dimensions that can be explored in this type of study.

Besides the theoretical aspect, the article list questions that can be used as starting points in an investigation of this audit modality, besides practical examples of situations in which the types of flaws that management audit can evidence and help to eliminate.
Revista do

Serviço

Público

Ano 54

Número 4

Out-Dez, 200

Carlos Alberto

Sampaio de

Freitas é especialista em Avaliação de Políticas

Públicas pela Universidade do Rio de Janeiro (UFRJ) e Analista de Finanças e Controle do Tribunal de Contas da União. 